\title{
Incidental findings in clinical genomics: a clarification
}

\author{
American College of Medical Genetics and Genomics
}

\begin{abstract}
Disclaimer: This guideline is designed primarily as an educational resource for clinical and laboratory geneticists to help them provide quality medical services. Adherence to this guideline is completely voluntary and does not necessarily ensure a successful medical outcome. This guideline should not be considered inclusive of all proper procedures and tests or exclusive of other procedures and tests that are reasonably directed to obtaining the same results. In determining the propriety of any specific procedure or test, the geneticist should apply his or her own professional judgment to the specific clinical circumstances presented by the individual patient or specimen.

Clinical and laboratory geneticists are encouraged to document the reasons for the use of a particular procedure or test, whether or not it is in conformance with this guideline. They also are advised to take notice of the date this guideline was adopted and to consider other medical and scientific information that becomes available after that date. It also would be prudent to consider whether intellectual property interests may restrict the performance of certain tests and other procedures.
\end{abstract}

On 22 March 2013, the American College of Medical Genetics and Genomics (ACMG) released a practice statement entitled "ACMG recommendations for reporting of incidental findings in clinical exome and genome sequencing." We firmly stated our view that there is a subset of conditions/genes/variants for which there is the significant potential for preventing disease morbidity and mortality if identified in the presymptomatic period. Commentaries about the ACMG recommendations have raised a number of concerns that prompt us to clarify five issues raised in the recommendations: (i) patient autonomy, (ii) incidental findings in children, (iii) clinical laboratory considerations, (iv) result communication, and (v) prediction of disease likelihood.

\section{PATIENT AUTONOMY}

The ACMG statement recommends that laboratories performing clinical genome (or exome) sequencing examine the sequence data generated from a set of 57 carefully chosen genes for pathogenic mutations that could indicate the presence of any of 24 disorders for which early intervention is likely to reduce or prevent serious morbidity or early mortality. It recommends that pretest counseling include a discussion of possible incidental findings, with the understanding that patients cannot opt out of the laboratory's reporting of incidental findings to the ordering clinician. It is expected that the clinician will contextualize these findings to the clinical circumstances (e.g., the nature of ongoing clinical problems, knowledge of personal and family history, patient preferences), and the provider and patient will participate in a shared decision-making process regarding the return of results. This is similar to the shared decision making that is undertaken by patients and physicians: whenever complex medical testing is contemplated, patients are informed that data generated from such tests can reveal unexpected results.

The rationale for our recommendations was that failure to report a laboratory test result conveying the near certainty of an adverse yet potentially preventable medical outcome would be unethical. A common objection to this recommendation has been that some genetic test results might not be predictive of disease, either because of incomplete penetrance or because of the occurrence of variants of unknown significance. The recommendations, however, explicitly focus only on unequivocally pathogenic mutations in genes in which pathogenic variants lead to disease with very high probability and cases in which evidence strongly supports the benefits of early intervention. We agree that variants of unknown significance, variants associated with low or unknown penetrance, and variants associated with disorders not currently amenable to intervention should not be reported.

\section{INCIDENTAL FINDINGS IN CHILDREN}

Some commentators have argued that our recommendations change long-standing policies about genetic testing in children. The ACMG has reaffirmed in a recent joint statement with the American Academy of Pediatrics ${ }^{2}$ that diagnostic genetic testing should be "driven by the best interests of the child" and that carrier screening and presymptomatic testing of children at risk for adult-onset diseases should be deferred until the child reaches maturity. Genome sequencing in children should be ordered and performed as a diagnostic test only if there are clear clinical indications. Some incidental findings would clearly benefit the child; for example, finding a pathogenic RET gene mutation that predicts risk of medullary thyroid carcinoma that can occur during childhood. Finding a variant that predicts an

${ }^{1}$ American College of Medical Genetics and Genomics, Bethesda, Maryland, USA. Approved by the ACMG Board of Directors, 25 April 2013. Correspondence: Michael Watson (mwatson@acmg.net) 
adult-onset condition, such as a BRCA1 gene mutation, however, would seem to violate the precept of testing children only for their immediate medical benefit.

We believe, however, that the disclosure of incidental findings such as a BRCA1 gene mutation is justified for the following reasons. (i) If the child carries a pathogenic mutation, there is a high probability that one parent does as well. Given that this is an incidental finding, it is fair to assume that the presence of this variant in the family has not been previously recognized based on clinical findings or family history. In this circumstance, and because only medically actionable variants highly likely to be pathogenic would be reported, the child does benefit by potentially preventing a severe adverse health outcome in a parent. (ii) The recommendation that children not be tested for an adult-onset disorder is typically invoked in circumstances in which there is a known family history of risk, with the expectation that the child will be offered testing at an age when he or she can make an informed decision about testing. If there are no other clinical or family history indications, as might be the case for an incidental finding, that opportunity may not occur, potentially until the child is affected. (iii) There is also some concern that the nurturing of the child might be adversely affected by the parent's knowledge of the child's future risk and the need to decide when to reveal that to the child. We believe, however, that the ability to identify a significant medical risk for the child that could avoid future morbidity takes precedence over this possible risk. The ACMG affirms its recommendation not to perform diagnostic testing for an adult-onset condition in children but believes that reporting an incidental finding of a severe, actionable, pathogenic mutation falls outside this recommendation.

\section{CLINICAL LABORATORY CONSIDERATIONS}

The ACMG has previously articulated the position that a laboratory should have a clear policy on whether it reports incidental findings resulting from genome sequencing. ${ }^{3}$ The current recommendation defines a minimal set of incidental findings that we believe should be sought and reported by the laboratory. Indeed, given the low prior probability of an individual having such an incidental finding, it is imperative that a very high bar be set with return of only those variants with a very high probability of being deleterious. Otherwise, the risk of false-positives will be significant. We recognize that some exome- or genome-sequencing tests may not be optimized for coverage of variants associated with these incidental findings. We do not recommend that laboratories modify these tests if they are otherwise suitable to achieve their clinical objectives; in such cases, however, laboratories should specify that the test was not optimized to detect incidental findings.

\section{RESULT COMMUNICATION}

Laboratory tests are ordered by clinicians based on the medical needs of their patients, and the results are typically returned to the referring clinician. Only recently have laboratories been either encouraged or required to make results directly available to patients. Patients who seek out their laboratory test results independent of their health-care providers have made their own choice about learning these results. The ACMG has published a policy statement ${ }^{4}$ expressing strong concerns regarding direct-to-consumer testing, recommending that a knowledgeable professional be involved in ordering and interpreting genetic test results. Physicians often have to deal with clinical situations outside of their primary area of practice, so consultation with appropriate specialists (e.g., clinical geneticists) to integrate genomic data with the patient's clinical situation and family history is optimal. The ACMG is currently developing a set of clinical decision support tools referred to as "ACT Sheets" to guide referring physicians through what may be their first encounter with genomic medicine.

\section{PREDICTING DISEASE LIKELIHOOD}

The ACMG recognizes that there is much to be learned about the prediction of disease from genomic testing in asymptomatic individuals. For this reason, the list of conditions for which it is recommended that incidental findings be reported is limited to conditions and variants where there is strong evidence of high probability of severe adverse medical outcomes that can be prevented or ameliorated by existing modalities. We expect that this list will evolve as more information is acquired about these and other diseases/variants. The principle of returning incidental findings, however, transcends the individual conditions on the list. There is a great need to develop a central repository of genotypic and phenotypic data to further inform this process. In the coming months, the ACMG will develop an open and transparent means by which the community can offer input into the further curation of the list of recommended conditions, genes, and variants.

\section{CONCLUSION}

The era of genomic medicine has begun, and we expect that it will continue to challenge long-held models of medical practice. The ACMG recommendations on return of incidental findings resulting from genome sequencing represent an early step in responding to this challenge. Many issues remain to be addressed, such as billing and reimbursement for testing that includes incidental finding identification and the approach to incidental findings identified in family members who are tested to help interpret the results in a patient. We appreciate the constructive dialogue that our statement has generated and look forward to working with the medical community and the public to ensure the best and most ethical use of genomic information in medical decision making going forward.

\section{DISCLOSURE}

The author declares no conflict of interest. 


\section{REFERENCES}

1. Green RC, Berg JS, Grody WW, et al. ACMG recommendations for reporting of incidental findings in clinical exome and genome sequencing. Genet Med 2013;15:565-574 (http://www.acmg.net/docs/ACMG_Releases_ Recommendations_on_Incidental_Findings_in_Clinical_Exome_and_ Genome_Sequencing.pdf)

2. Ross LF, Saal HM, David KL, Anderson RR; the American Academy of Pediatrics, and American College of Medical Genetics and Genomics. Technical report: ethical and policy issues in genetic testing and screening of children. Genet Med 2013;15: 234-245.

3. ACMG. ACMG statement on direct-to-consumer genetic testing. http://www acmg.net/AM/Template.cfm?Section=Policy_Statements\&Template=/CM/ ContentDisplay.cfm\&ContentID=2975. 7 April, 2008.

4. ACMG. ACMG statement on points to consider in the clinical application of genomic sequencing. (2012) (http://www.acmg.net/StaticContent/PPG/ Clinical_Application_of_Genomic_Sequencing.pdf) Genet Med 2012;14: 759-761. doi:10.1038/gim.2012.74. 